\title{
Surface plasmon excitations in metal spheres: Direct comparison of light scattering and electron energy-loss spectroscopy by modal decomposition
}

\author{
Sean M. Collins* and Paul A. Midgley ${ }^{\dagger}$ \\ Department of Materials Science and Metallurgy, \\ University of Cambridge, Pembroke Street, \\ Cambridge CB2 3QZ, United Kingdom
}

(Dated: June 30, 2013)

\begin{abstract}
In previous publications, qualitative agreement between studies of surface plasmon excitations in nanoparticles by near field light scattering and electron energy-loss spectroscopy (EELS) has been found for experiments and simulations. Here, we present a quantitative method for the comparison of light scattering and EELS for surface plasmons in metal spheres. Defining the Fourier transform of the modal component of the scattered electric field along the equivalent electron trajectory enables a direct evaluation of the relative weighting factor for light- and electron-excited surface plasmon modes. This common quantity for light scattering and EELS is examined for size, composition, and trajectory dependencies, facilitating the analysis of key differences between light and electron excitation. A single functional dependence on Drude model plasmon energies is identified to explain the relative modal weighting factors for light scattering and EELS. This method represents an important step toward the complete spectral and spatial reconstruction of EELS maps from near field light scattering calculations.
\end{abstract}




\section{INTRODUCTION}

Investigations of the optical modes of plasmonic nanostructures by electron energy-loss spectroscopy (EELS) in the transmission electron microscope (TEM) have been realized recently following instrumental developments in electron beam monochromation ${ }^{1-3}$ and a growing interest in developing plasmonic nanostructures for such applications as sensing and spectroscopy, ${ }^{4,5}$ solar energy conversion and storage, ${ }^{6,7}$ lighting, ${ }^{8,9}$ catalysis, ${ }^{10,11}$ and nanophotonic devices. ${ }^{12,13}$ EELS in the TEM allows for the examination of plasmonic modes at nanometer resolution, well below the diffraction limit of visible light. As such, the information content of EELS signals recorded in the TEM corresponds to a near field response of plasmonic nanostructures. Since it is likewise a near field response that enables the unique light scattering (LS) behavior of plasmonic technologies, EELS is seemingly well-suited to the study of plasmonic nanostructures.

However, EELS spectra and maps do not capture the same information as in LS, due to differences in plasmon excitations by the evanescent field of a swift electron and the timevarying dipolar field of light. Several types of dark modes excited by electron beams have been reported that are not generally accessible with light excitation. ${ }^{14-16}$ For those modes that are excited both by electrons and light, a number of approaches for the qualitative comparison of LS simulations and experimental and simulated EELS signals have been reported. Commonly, the modulus squared of the electric field, $|\mathbf{E}|^{2}$, or its component along the electron trajectory, $\left|E_{z}\right|^{2}$, is plotted at a plane some distance above the particle. ${ }^{17,18}$ Alternatively, far field LS spectra and $|\mathbf{E}|$ have been compared to EELS ${ }^{19,20}$ although far field spectra are known to exhibit energy offsets in peak maxima in comparison with the near

field response. ${ }^{21}$ Several strategies have been applied including multiple light polarizations in the calculation of $\left|E_{z}\right|^{2}$ in order to reproduce EELS signals. ${ }^{17,22,23}$ Many of these approaches yield satisfactory qualitative comparisons between plasmon modes observed in EELS and LS near fields. Correspondence between EELS and potential at a plane above the particle has also been reported. ${ }^{24}$ For translationally symmetric or thin particles, the electromagnetic local density of states (EMLDOS) at a plane some distance above the particle has in fact been demonstrated to correspond well to the EELS signal. ${ }^{25}$ The comparison with the EMLDOS is not universal, though, and several distinctions between the EMLDOS and EELS signals have been reported. ${ }^{24}$ 
Moreover, comparisons with $\left|E_{z}\right|^{2}$ and the EMLDOS do not convey the interaction of the electron beam and the particle. These comparisons are plotted typically at a plane some distance from the particle, and do not directly represent the trajectory of the electron. Recent advances in the simulation of plasmon EELS have elaborated the electron-excited near field response ${ }^{26-28}$ and identified similarities in light- and electron-induced fields. ${ }^{29}$ However, a quantitative tool for directly relating EELS and LS of plasmonic nanoparticles has not yet been reported.

As a means for understanding EELS and scanning near field optical microscopy (SNOM) signals, Boudarham and Kociak recently reported on modal decompositions of the induced charge density, potential, and electric field in the non-retarded limit. ${ }^{30}$ The modal decomposition approach offers general expressions for distinguishing the interaction recorded in these two different microscopies and has been applied to simulating the EELS and SNOM signals for plasmonic nanorods. ${ }^{30}$ The EMLDOS has also been compared at a plane above the particle ${ }^{30}$ consistent with previous work. ${ }^{25}$ The correspondence of EELS probabilities to decomposed modal field or potential contributions ${ }^{30}$ further underscores challenges in comparing total fields or total potentials for EELS and LS.

Here, we demonstrate the tenability of extending the modal decomposition approach to comparing LS to EELS for isolated spheres. Boudarham and Kociak define the EELS signal for non-penetrating electron trajectories in terms of the Fourier transform of the modal electric field along the trajectory. ${ }^{30}$ Using fully relativistic and retarded expressions for the EELS probability and LS near a sphere, we now present a comparison of EELS and LS within this suggested formalism of a Fourier transform of the modal scattered field along the direction of propagation. Following comparison of the analytical solutions for EELS and LS, the systematic dependencies on composition, size, and impact parameter are examined. The success of this approach represents an initial step in relating LS and EELS signals in a quantitative manner.

\section{SURFACE PLASMON MODES OF A SPHERE}

The energy loss of a swift electron $\Delta E$ due to surface plasmon excitation may be described as the work done on the electron by the induced or scattered electric field acting back on 
the electron. ${ }^{31,32}$ This relationship establishes a connection to the loss probability $\Gamma^{E E L S}$ :

$$
\Delta E=\int_{-\infty}^{\infty} d t q_{e}\left(\mathbf{v} \cdot \mathbf{E}_{\mathbf{s c a}}[\mathbf{r}(t), t]\right)=\int_{0}^{\infty} d \omega \hbar \omega \Gamma^{E E L S}(\omega)
$$

where the electron has charge $q_{e}$, traveling with constant velocity $\mathbf{v}$ along a straight line trajectory $\mathbf{r}=\left(\mathbf{R}_{\mathbf{0}}, z\right)$, and $\mathbf{E}_{\mathbf{s c a}}$ is the scattered field. Given $\mathbf{E}_{\mathbf{s c a}}[\mathbf{r}, \omega]=\mathbf{E}_{\mathbf{s c a}}[\mathbf{r}, \omega]^{*}$ this expression can be further simplified by Fourier transformation of $\mathbf{E}_{\mathbf{s c a}}[\mathbf{r}, t]$ to yield

$$
\Gamma^{E E L S}(\omega)=\frac{q_{e}}{\pi \hbar \omega} \int_{-\infty}^{\infty} d t \Re\left\{e^{-i \omega t}\left(\mathbf{v} \cdot \mathbf{E}_{\mathbf{s c a}}[\mathbf{r}(t), \omega]\right)\right\}
$$

Following Zabala and Rivacoba, ${ }^{33}$ in the frequency domain the surface plasmon energy-loss probability $\Gamma^{\text {surf }}$ in the non-retarded case can in turn be written as

$$
\Gamma^{\text {surf }}\left(\mathbf{R}_{\mathbf{0}}, \omega\right)=-\frac{1}{\pi} \int_{-\infty}^{\infty} d z \int_{-\infty}^{\infty} d z^{\prime} \Im\left\{\rho\left(\mathbf{R}_{\mathbf{0}}, z, \omega\right)^{*} G^{\text {surf }}\left(\mathbf{R}_{\mathbf{0}}, z, \mathbf{R}_{\mathbf{0}}, z^{\prime}, \omega\right) \rho\left(\mathbf{R}_{\mathbf{0}}, z^{\prime}, \omega\right)\right\}
$$

where $\rho$ is the charge distribution of the electron beam, $G^{\text {surf } f}$ is the scalar Green's function, and $\mathbf{R}_{\mathbf{0}}=(x, y)$. The charge distribution of the electron beam is commonly given as $\rho\left(\mathbf{R}_{\mathbf{0}}, z, t\right)=-q_{e} \delta\left(\mathbf{R}-\mathbf{R}_{\mathbf{0}}\right) \delta(z-v t)$ and its Fourier transform is ${ }^{24,30}$

$$
\rho\left(\mathbf{R}_{\mathbf{0}}, z, \omega\right)=-q_{e} \delta\left(\mathbf{R}-\mathbf{R}_{\mathbf{0}}\right) \frac{e^{i z \omega / v}}{v} .
$$

By using a modal decomposition of the induced potential, Boudarham and Kociak have demonstrated that for non-penetrating electron trajectories, the energy-loss probability in terms of potential $\phi$ becomes ${ }^{30}$

$$
\Gamma^{\text {surf }}\left(\mathbf{R}_{\mathbf{0}}, \frac{\omega}{v}\right)=-\frac{1}{\pi v^{2}} \sum_{l} \Im\left\{g_{l}(\omega)-\frac{1}{\epsilon_{2}(\omega)}\right\}\left|\phi^{\text {surf }, l}\left(\mathbf{R}_{\mathbf{0}}, \frac{\omega}{v}\right)\right|^{2},
$$

where $g_{l}(\omega)$ are modal weighting factors and $\epsilon_{2}(\omega)$ is the dielectric function of the medium surrounding the particle. The corresponding electric field and its Fourier transform are

$$
\begin{aligned}
E_{z}^{s u r f, l}\left(\mathbf{R}_{\mathbf{0}}, z\right) & =-\frac{\partial}{\partial z} \phi^{s u r f, l}\left(\mathbf{R}_{\mathbf{0}}, z\right) \\
E_{z}^{s u r f, l}\left(\mathbf{R}_{\mathbf{0}}, \frac{\omega}{v}\right) & =-i \frac{\omega}{v} \phi^{s u r f, l}\left(\mathbf{R}_{\mathbf{0}}, \frac{\omega}{v}\right) .
\end{aligned}
$$

Consequently, the total EELS probability due to surface plasmon excitation is

$$
\Gamma^{E E L S}\left(\mathbf{R}_{\mathbf{0}}, \omega\right)=\frac{1}{\pi \omega^{2}} \sum_{l} \Im\left\{-g_{l}(\omega)\right\}\left|E_{z}^{\operatorname{surf}, l}\left(\mathbf{R}_{\mathbf{0}}, \frac{\omega}{v}\right)\right|^{2}
$$


For simple geometries including spheres and infinite cylinders, analytical solutions to the Poisson equation have been reported previously and likewise consist of a sum over modes. In the case of a sphere, the electron energy-loss probability for non-penetrating trajectories is given $b^{34,35}$

$$
\Gamma^{E E L S}(b, \omega)=\frac{4 a}{\pi v^{2}} \sum_{l=1}^{\infty} \sum_{m=0}^{l} \frac{2-\delta_{m 0}}{(l+m) !(l-m) !} \Im\left\{\alpha_{l}(\omega)\right\}\left[\frac{\omega a}{v}\right]^{2 l} K_{m}^{2}\left(\frac{\omega b}{v}\right),
$$

where $K_{m}$ is a modified Bessel function of the second kind, $\delta_{m 0}$ is the Kronecker delta function, $a$ is the sphere radius, $b$ is the impact parameter as illustrated in Fig. 1, and $\alpha_{l}(\omega)$ are sphere response functions defined in terms of the complex dielectric function of the particle, $\epsilon$, by

$$
\alpha_{l}(\omega)=\frac{l(1-\epsilon)}{l \epsilon+l+1}
$$

A similar modal sum describes the fully relativistic and retarded solution to Maxwell's equations for external electron excitation. ${ }^{32,36}$ Following the notation in Ref. 36, we define the EELS probability as

$$
\Gamma^{E E L S}(b, \omega)=\frac{1}{\omega} \sum_{l=1}^{\infty} \sum_{m=-l}^{l}\left[C_{l m}^{E E L S, a} \Im\left\{i a_{l}\right\}+C_{l m}^{E E L S, b} \Im\left\{i b_{l}\right\}\right],
$$

where $a_{l}$ and $b_{l}$ are the electric and magnetic Mie expansion coefficients, respectively, given as

$$
\begin{aligned}
a_{l} & =\frac{\epsilon j_{l}\left(x_{2}\right)\left[x_{1} j_{l}\left(x_{1}\right)\right]^{\prime}-j_{l}\left(x_{1}\right)\left[x_{2} j_{l}\left(x_{2}\right)\right]^{\prime}}{\epsilon\left[x_{1} h_{l}^{(1)}\left(x_{1}\right)\right]^{\prime} j_{l}\left(x_{2}\right)-h_{l}^{(1)}\left(x_{1}\right)\left[x_{2} j_{l}\left(x_{2}\right)\right]^{\prime}} \\
b_{l} & =\frac{j_{l}\left(x_{2}\right)\left[x_{1} j_{l}\left(x_{1}\right)\right]^{\prime}-j_{l}\left(x_{1}\right)\left[x_{2} j_{l}\left(x_{2}\right)\right]^{\prime}}{\left[x_{1} h_{l}^{(1)}\left(x_{1}\right)\right]^{\prime} j_{l}\left(x_{2}\right)-h_{l}^{(1)}\left(x_{1}\right)\left[x_{2} j_{l}\left(x_{2}\right)\right]^{\prime}} .
\end{aligned}
$$

Here $x_{1}=k a, x_{2}=k a \sqrt{\epsilon}, k=2 \pi / \lambda$ is the wavenumber, and $j_{l}$ and $h_{l}^{(1)}$ are spherical Bessel functions and spherical Hankel functions, respectively. Primes denote derivatives with respect to the argument $x_{1}$ or $x_{2}$. The EELS excitation introduces coefficients $C^{E E L S, a}$ and $C^{E E L S, b}$ given as

$$
\begin{aligned}
C_{l m}^{E E L S, a} & =K_{m}^{2}\left(\frac{\omega b}{v \gamma}\right) \frac{1}{l(l+1)}\left|2 m N_{l m}\right|^{2} \\
C_{l m}^{E E L S, b} & =K_{m}^{2}\left(\frac{\omega b}{v \gamma}\right) \frac{1}{l(l+1)}\left|\frac{c}{v \gamma} M_{l m}\right|^{2},
\end{aligned}
$$


where $\gamma$ is the Lorentz contraction factor, $\gamma=1 / \sqrt{1-v^{2} / c^{2}}$, and $N_{l m}$ and $M_{l m}$ are given in terms of Gegenbauer polynomials $G_{n}^{u}$ :

$$
\begin{aligned}
N_{l m} & =\sqrt{\frac{(2 l+1)}{\pi} \frac{(l-|m|) !}{(l+|m|) !} \frac{(2|m|-1) ! !}{(v \gamma / c)} G_{l-|m|}^{|m|+1 / 2}\left(\frac{c}{v}\right),} \\
M_{l m} & =N_{l m+1} \sqrt{(l+m+1)(l-m)}+N_{l m-1} \sqrt{(l-m+1)(l+m)} .
\end{aligned}
$$

The retarded expression for the EELS probability is given in terms of the same Mie expansion coefficients as the scattered field resulting from a plane wave incident on a sphere. The decomposition in both EELS and LS analytical expressions is therefore defined over the same modes. Specifically, the z-component of the electric field induced by a plane wave of light can be written in spherical coordinates $(r, \theta, \phi) \mathrm{as}^{37}$

$$
E_{z}(r, \theta, \phi, \omega)=\sum_{l=1}^{\infty}\left[C_{l}^{L S, a} i a_{l}+C_{l}^{L S, b} b_{l}\right],
$$

where $a_{l}$ and $b_{l}$ are again the Mie expansion coefficients given in Eq. 12-13 and the corresponding light scattering coefficients $C^{L S, a}$ and $C^{L S, b}$ are given by

$$
\begin{aligned}
C_{l}^{L S, a} & =E_{l} \frac{\sin \theta \cos \phi}{k r}\left[l(l+1) h_{l}^{(1)}(k r) \pi_{l} \cos \theta-\xi_{l}^{\prime} \tau_{l}\right] \\
C_{l}^{L S, b} & =E_{l} \frac{\sin \theta \cos \phi}{k r} \xi_{l} \pi_{l},
\end{aligned}
$$

where the modal field coefficient derived from the expansion of a plane wave in spherical harmonics, $E_{l}$, and the Ricatti-Bessel function $\xi_{l}$ are given by

$$
\begin{aligned}
E_{l} & =\frac{i^{l} E_{0}(2 l+1)}{l(l+1)} \\
\xi_{l} & =k r h_{l}^{(1)}(k r) .
\end{aligned}
$$

Here $E_{0}$ is the amplitude of the incident plane wave. The angular functions $\pi_{l}$ and $\tau_{l}$ are given by the initial values and recurrence relations:

$$
\begin{aligned}
\pi_{0} & =0 \\
\pi_{1} & =1 \\
\pi_{l} & =\frac{2 l-1}{l-1}(\cos \theta) \pi_{l-1}-\frac{l}{l-1} \pi_{l-2} \\
\tau_{l} & =l(\cos \theta) \pi_{l}-(l+1) \pi_{l-1} .
\end{aligned}
$$

In order to directly compare LS and EELS, the LS near field can be brought into a form compatible with the EELS probability. Because the electron probes along its trajectory and, 
more precisely, loses energy with a probability proportional to the Fourier transform of the electric field (Eq. 8), a new quantity can be defined to translate the LS near field into a form analogous to the EELS probability. This quantity will be referred to here likewise as a probability for the purpose of comparison with the EELS probability. It does not itself represent a physical probability in LS but provides a convenient means for quantitatively comparing the distinct excitation signals. The transformed LS probability is defined by inserting the LS electric field for a particular mode $l$ into the equation for the EELS probability given in terms of electric field (Eq. 8):

$$
\Gamma_{l}^{L S}=\frac{1}{\pi \omega^{2}}\left|\int_{-\infty}^{\infty} d z e^{-i \omega z / v} E_{z}^{L S, l}\left(\mathbf{R}_{\mathbf{0}}, z, \omega\right)\right|^{2} .
$$

Here, Eq. 8 has been rewritten in the form:

$$
\begin{aligned}
\Gamma_{l}^{E E L S} & =\frac{1}{\pi \omega^{2}} \Im\left\{-g_{l}(\omega)\right\}\left|\int_{-\infty}^{\infty} d z e^{-i \omega z / v} E_{z}^{E E L S, l}\left(\mathbf{R}_{\mathbf{0}}, z\right)\right|^{2} \\
& =\frac{1}{\pi \omega^{2}}\left|\int_{-\infty}^{\infty} d z e^{-i \omega z / v}\left[\Im\left\{-g_{l}(\omega)\right\}\right]^{1 / 2} E_{z}^{E E L S, l}\left(\mathbf{R}_{\mathbf{0}}, z\right)\right|^{2} \\
& =\frac{1}{\pi \omega^{2}}\left|\int_{-\infty}^{\infty} d z e^{-i \omega z / v} E_{z}^{E E L S, l}\left(\mathbf{R}_{\mathbf{0}}, z, \omega\right)\right|^{2},
\end{aligned}
$$

where

$$
E_{z}^{E E L S, l}\left(\mathbf{R}_{\mathbf{0}}, z, \omega\right)=\left[\Im\left\{-g_{l}(\omega)\right\}\right]^{1 / 2} E_{z}^{E E L S, l}\left(\mathbf{R}_{\mathbf{0}}, z\right)
$$

The definition of the transformed LS probability in Eq. 27 is directly comparable with Eq. 28c, distinguished by the respective LS or EELS field $E_{z}^{l}\left(\mathbf{R}_{\mathbf{0}}, z, \omega\right)$. Insertion of the analytical solution to Maxwell's equations for the LS field of a sphere for a particular mode $l$ (Eq. 18) gives

$$
\Gamma_{l}^{L S}=\frac{1}{\omega^{2}}\left|\int_{-\infty}^{\infty} d z e^{-i \omega z / v}\left[C_{l}^{L S, a} i a_{l}+C_{l}^{L S, b} b_{l}\right]\right|^{2}
$$

A change from the spherical coordinates $(r, \theta, \phi, \omega)$ of Eq. 18 to Cartesian coordinates $\left(\mathbf{R}_{\mathbf{0}}, z, \omega\right)$ is implicit in the symbolic notation in Eq. 30.

Further, the analytical EELS probability given by Eq. 11 is substituted for the expression for the EELS probability in terms of electric field given in Eq. 8 or the equivalent Eq. 28c. 
For a particular mode $l$, the transformed LS and EELS probabilities are then given as:

$$
\begin{gathered}
\Gamma_{l}^{L S}(b, \omega)=\omega^{-2}\left|F_{\omega / v}\left\{C_{l}^{L S, a}\right\} i a_{l}+F_{\omega / v}\left\{C_{l}^{L S, b}\right\} b_{l}\right|^{2}, \\
\Gamma_{l}^{E E L S}(b, \omega)=\omega^{-1} \sum_{m=-l}^{l}\left[C_{l m}^{E E L S, a} \Im\left\{i a_{l}\right\}+C_{l m}^{E E L S, b} \Im\left\{i b_{l}\right\}\right] .
\end{gathered}
$$

Here $F_{\omega / v}$ denotes the Fourier transform with respect to $z$ as given in Eq. 27-30. In the approximation that electric modes dominate, which is true for plasmonic metals commonly investigated by EELS and LS, the magnetic terms may be eliminated resulting in the comparison:

$$
\begin{aligned}
\Gamma_{l}^{L S}(b, \omega) & =\chi_{l}^{L S}\left|i a_{l}\right|^{2}, \\
\Gamma_{l}^{E E L S}(b, \omega) & =\chi_{l}^{E E L S} \Im\left\{i a_{l}\right\},
\end{aligned}
$$

where now the coefficients are rewritten in the form:

$$
\begin{aligned}
\chi_{l}^{L S} & =\frac{1}{\omega^{2}}\left|F_{\omega / v}\left\{C_{l}^{L S, a}\right\}\right|^{2} \\
\chi_{l}^{E E L S} & =\frac{1}{\omega} \sum_{m=-l}^{l} C_{l m}^{E E L S, a} .
\end{aligned}
$$

For both EELS and transformed LS probabilities the modal maxima are given by the zeros of the denominator of the Mie expansion coefficient. ${ }^{38}$ For the case of surface plasmons in metals, where the dielectric function is dominated by a negative real part, $\Im\left\{i a_{l}\right\} \approx\left|i a_{l}\right|$ and consequently the same modes appear in transformed LS and EELS probabilities. The Mie expansion coefficients in fact give modes defined only by the particle geometry, as dictated by the sphere radius, and composition, given by the dielectric function. The remaining terms determine the relative weight of each mode but depend only on the physical parameters of the excitation, the impact parameter and velocity.

The modes given by the Mie expansion coefficients are not identical to the eigenmodes proposed by Boudarham and Kociak as the geometric eigenmodes proposed in the nonretarded case are independent of composition and size. ${ }^{30}$ However, the fundamental modes of a sphere determined by solutions to Maxwell's equations are required in order to account for sizeable retardation effects, ${ }^{36}$ and these modes provide the best analogy to the nonretarded modes determined from solutions to the Poisson equation. ${ }^{30}$ 


\section{COMPUTATIONAL METHODS}

In order to evaluate the outlined comparison of EELS and LS and to examine the roles of composition, size, and impact parameter, Eq. 31-32 were computed numerically. Eq. 32 was calculated using a Matlab function implementation following Ref. 32 and 36. Difficulties in evaluating the Fourier transform of spherical terms in Eq. 31 were readily avoided as the transformation was approximated by numerical evaluation of discretized Mie light scattering near field calculations. Such LS calculations were performed using near field Mie scattering codes adapted from MatScat, ${ }^{39}$ a publicly available Matlab project based on programs by Bohren and Huffman. ${ }^{37}$ For comparison to EELS, the near field was transformed according to Eq. 30-31. Convergence of the definite integrals used to approximate the Fourier transform in Eq. 30 was confirmed for all presented calculations. Integration limits of $\pm 8 a$ and a step size $d z \approx \Delta z=0.04 a$ were typically sufficient. The decay of the scattered electric field (Eq. 27) away from the sphere surface predominantly determined convergence requirements. For example, for a $100 \mathrm{~nm} \mathrm{Al}$ sphere and $l=1, \Re\left\{E_{z}\right\}$ and $\Im\left\{E_{z}\right\}$ at the limits of integration were each $<2 \%$ of the maximal values. Consequently, the integrand contributed minimally beyond these limits. Evaluation of extended integration limits and finer step sizes yielded peaks with energy positions consistent within the energy step size of $0.01 \mathrm{eV}$ and absolute

intensities within $6 \%$ at $b=1.1 a$. For both LS and EELS, modes $l \leq 15$ were calculated to adequately represent the total EELS probability. ${ }^{36}$

For LS calculations, the polarization of the plane wave was selected to coincide with the predominant electric field direction toward the electron in a corresponding EELS configuration. Comparisons of LS and EELS induced fields have previously demonstrated the validity of such selection of the light polarization. ${ }^{29}$ In both LS and EELS calculations, Drude model dielectric functions of the form

$$
\epsilon(\omega)=1-\frac{\omega_{P}^{2}}{\omega(\omega+i \Gamma)}
$$

were used to model the response of plasmonic metal spheres. Here $\omega_{P}$ is the plasmon frequency and $\Gamma$ is an internal damping parameter. 


\section{RESULTS}

Figure 2 presents a demonstration of the precise match between individual modes in EELS and transformed LS for the case of an $\mathrm{Al}$ sphere $(10 \mathrm{~nm}$ diameter, $b=1.1 a)$. In each case, LS modes have been scaled to the maximum in the respective EELS mode and normalized to the peak in the EELS probability $\Gamma^{\max }$. The total EELS probability is shown for reference. Individual transformed LS and EELS modes are closely matched in position, line width, and line shape.

The well-matched modal characters recovered from transformed LS are not represented in the more common comparison of EELS with $|\mathbf{E}|^{2}$ or $\left|E_{z}\right|^{2}$ at a plane above the particle. Figure 3(a)-(c) illustrates the key difficulty in comparing EELS signals to $\left|E_{z}\right|^{2}$ at a plane above a sphere for a $100 \mathrm{~nm}$ diameter $\mathrm{Al}$ sphere $(b=1.1 a)$, a case where differences are pronounced. The line shape is distorted as a function of energy, and moreover, the peak position is not a constant function of distance above the particle. In Fig. 3(c), transformed LS recovers the EELS line shape (Fig. 3(b)) and yields a single peak position. Selection of a plane above a particle for comparison with point dipole excitation has been shown to bear qualitative similarity to EELS for potential and fields because the spatial decays have similar

functional forms to the LDOS. ${ }^{24,30}$ The selection of a particular plane, however, cannot be optimized $^{24}$ and comparisons are therefore qualitative only. For plane wave light excitation, qualitative analogy between the field at a plane near the particle and EELS may be possible but does not provide a method for consistent quantitative comparison with EELS.

An alternative simple comparison of LS to EELS is the projection of a physical property along the trajectory. In the limit of small $\omega z / v$, the Fourier transform in Eq. 30 approaches an integral along the trajectory. Figure 4 presents an evaluation of this approximation for (a) electric field and (b) electric potential. Possible approximations are given explicitly as

$$
\begin{aligned}
& \Gamma_{l} \approx\left|\int_{-\infty}^{\infty} d z E_{z}^{l}\left(\mathbf{R}_{\mathbf{0}}, z\right)\right|^{2}, \\
& \Gamma_{l} \approx \frac{1}{\omega^{2}}\left|\int_{-\infty}^{\infty} d z E_{z}^{l}\left(\mathbf{R}_{\mathbf{0}}, z\right)\right|^{2},
\end{aligned}
$$


and

$$
\Gamma_{l} \approx\left|\int_{-\infty}^{\infty} d z \phi^{l}\left(\mathbf{R}_{\mathbf{0}}, z\right)\right|^{2}
$$

where Eq. 38 gives a simple projection of the electric field, Eq. 39 is a modified projection of the electric field following Eq. 8, and Eq. 40 is a projection of electric potential. The Fourier transforms in terms of electric field and potential are given in Eq. 5-7. Figure 4(a) presents the projected electric field along the trajectory for modes $l=1-3$. The projection of $E_{z}$ is catastrophic particularly for odd modes $(l=2 n+1, n=0,1,2, \ldots)$. These odd modes consist of an odd number of nodes in the electric field and consequently have nearly equal contributions of opposite sign above and below the plane of the sphere. Even modes are not as affected because the associated fields are dominated by the field at the plane containing the center of the sphere. The modal Fourier transformation avoids these artifacts.

Figure 4(b) highlights the superior approximation of projecting the electric potential along the trajectory. For both even and odd modes, the integrated potential along the trajectory yields signals very similar to EELS or the Fourier transform of potential. Nevertheless, the Fourier transform of the potential along the trajectory yields greater accuracy in comparing LS and EELS.

By transforming LS for comparison with EELS, not only do individual modes correspond to those observed in EELS, but the relative weight of transformed LS modes varies smoothly and systematically for variation in composition, sphere size, and impact parameter. Figure 5(a) presents composition-dependent variation in the relative weighting of transformed LS modes and EELS modes. The relative weights of modes as a function of the mode number $l$ decay approximately exponentially. Fitting of the mode number dependence for several metals modeled by Drude dielectric functions revealed a consistent correlation between the rate of decay and the plasmon energy $\omega_{P}$. Functions of the form (const) $e^{-\alpha l}$ were used for fitting as consistent estimators of the rate of decay. The exact underlying functional form of the relative weighting factors may differ but the decay rates are captured well using exponential functions. Drude model parameters for $\mathrm{Al}, \mathrm{Ag}$, $\mathrm{Na}$, and $\mathrm{Cu}$ followed Ref. 32 and 40. Additional hypothetical dielectric functions were examined to evaluate trends in $\omega_{P}$.

For both $5 \mathrm{~nm}$ and $50 \mathrm{~nm}$ diameter spheres, the rate of decay varies monotonically and as a single, consistent function of $\omega_{P} b / c$ [see Fig. 5(b)]. As $\omega_{P} b / c$ tends toward zero, the 
rate of decay increases. At higher $\omega_{P} b / c$, the rate of decay falls off gradually. The functional form matches the anticipated ratio of coefficients $\chi_{l}$ given by Eq. 35-36, which determine energy $(\hbar \omega)$ and impact parameter $(b)$ dependencies for each mode (see also Fig. 8 and Sec. $\mathrm{V})$.

The size dependence of EELS and transformed LS is examined separately in Fig. 6 for Al spheres 10-300 nm in diameter ( $b=a$ following Ref. 35). Figure 6(a) depicts the sizedependent red-shifting of modes due to retardation. The red-shifting of modes follows the same functional form for EELS and transformed LS. The slight red-shifting apparent in EELS at the high energy accumulation point for large spheres (Fig. 6(a), left) is due to incomplete modeling of the total EELS probability by the cutoff established at $l=15$. For transformed LS (Fig. 6, center and right), the signal is plotted as $\Gamma^{L S} / a^{3}$ to adjust for the increasing excited volume for LS as a function of sphere size. Whereas the excited volume for EELS is a function of the electron velocity, ${ }^{32}$ the excited volume in LS increases with sphere size because the plane wave is of constant magnitude throughout the sphere.

Figure 6(b)-(d) details the contribution of each mode as a function of reduced radius, $a \omega_{l} / v$. The fully retarded EELS probability closely follows the trends reported for the nonretarded case. ${ }^{35}$ Here $\omega_{l}=\omega_{P} \sqrt{l /(2 l+1)}$ as for the non-retarded expression given in Eq. 9. ${ }^{35}$ The EELS modes are maximally excited at values of $a \omega_{l} / v \approx l$. Due to the noted increase in excited volume for LS, the transformed LS modes all increase monotonically as a function of $a \omega_{l} / v$. As a simple, qualitative correction, adjustment by the sphere volume yields the size dependence for transformed LS in Fig. 6(d). Once corrected for the excited volume, the size dependence resembles the functional form of the EELS probability in Fig. 6(b). The correspondence is not exact because the correction for the excited volume is a coarse approximation but does, however, point to the key difference in excited volume when comparing transformed LS and EELS.

This distinction in the nature of surface plasmon mode excitation in transformed LS and EELS is borne out further by trends in impact parameter dependence (see Fig. 7). For 10 $\mathrm{nm}$ diameter $\mathrm{Al}$ spheres, the normalized $l=1$ mode follows the identical impact parameter dependence for transformed LS and EELS [see Fig. 7(a)]. The $l=2$ mode is only weakly excited in LS and is near zero for a $10 \mathrm{~nm}$ diameter sphere. For $100 \mathrm{~nm}$ diameter $\mathrm{Al}$ spheres [see Fig. 7(b)], the impact parameter dependence of the $l=1$ mode differs in transformed LS and EELS. The transformed LS signal falls off more gradually than the EELS signal. 
The $l=2$ mode is again relatively weakly excited.

The difference in impact parameter dependence for $100 \mathrm{~nm}$ diameter spheres can be understood by examining the variation of relative impact parameter $(b / a)$ dependence with size, presented in Fig. 7(c). In transformed LS, the relative impact parameter dependence is identical regardless of size. In EELS, the rate of decay with increasing relative impact parameter is greater for $100 \mathrm{~nm}$ spheres than for $10 \mathrm{~nm}$ spheres. This trend can be explained by the difference in the excited volume for LS and EELS. For LS, the excitation is identical for relative impact parameters for any size. For EELS, the excited volume in larger spheres is smaller for the same relative impact parameters because the absolute impact parameter places the electron trajectory further from the sphere. This analysis suggests comparisons of LS and EELS are more straightforward for small spheres. The comparison is also viable for larger spheres, but the adjustment for the systematic variation in impact parameter must be taken into account.

The dependencies on sphere size and impact parameter for electron excitation have been noted in analyses of EELS of surface plasmons for many years, ${ }^{34,41}$ and the corresponding variations in the comparison with LS are therefore unsuprising. However, previous methods for comparing LS have not provided a single metric $\Gamma^{L S}$ for quantitative comparison. The presented method allows for scaling each mode in LS to the corresponding EELS signal for a particular set of physical parameters. Cumulatively, the trends in composition, size, and impact parameter establish the parameter space for the quantitative comparison of LS and EELS.

\section{DISCUSSION: LIMITATIONS AND APPLICATIONS}

Several characteristics of EELS and transformed LS of surface plasmons require careful consideration for broader application of the comparison, including the functional forms of the energy, spatial, and velocity dependent coefficients $\chi_{l}^{L S}$ and $\chi_{l}^{E E L S}$ in Eq. 35-36 and the role of the direction of light polarization relative to the excited particle.

For large spheres, the large red-shifts due to retardation place the modes given by the Mie expansion coefficients in spectral regions that also exhibit large gradients in the coefficients $\chi_{l}^{L S}$ and $\chi_{l}^{E E L S}$. Figure 8 plots the energy dependence of the $l=1$ coefficients for an impact parameter $b=30 \mathrm{~nm}$ and an electron velocity corresponding to $300 \mathrm{kV}$. Only the 
Bessel function corresponding to $m=l$ is included in $\chi_{l}^{E E L S}$ for simplicity as it is the main contribution to the coefficient. ${ }^{42}$ Both EELS and transformed LS coefficients tend toward large values at low energies and approach zero at high energies. For broadened and redshifted plasmon modes, the product of the coefficient $\chi_{l}$ and the Mie coefficient results in a shift of the modal maximum. This shift is readily understood by considering the effect of the coefficient $\chi_{l}$ on the modal peak given by the Mie coefficients: a maximum will occur for a particular mode where the derivative of the modal probability is zero. Without considering the coefficient $\chi_{l}$, these maxima are given by the zeros of the denominator of the Mie coefficients ${ }^{38}$ (see also Sec. II). For simplicity of notation these peaks in the Mie coefficients will be labeled here as $\mu_{l}$. Given the positive values of $\chi_{l}$ and $\mu_{l}$, the product of the the coefficient $\chi_{l}$ and a peak $\mu_{l}$ requires the respective derivatives to be of opposite sign: $0=\left[\chi_{l} \mu_{l}\right]^{\prime}=\chi_{l} \mu_{l}^{\prime}+\chi_{l}^{\prime} \mu_{l}$. The negative slope of the coefficient $\chi_{l}$ (Fig. 8) results in a shift of the modal peak maximum to lower energies where the slope of $\mu_{l}$ is positive.

This shift is problematic in the case of comparing plasmon EELS and transformed LS probabilities in that the magnitude of the shift is not consistent between EELS and LS. Moreover, this shift is not consistent for EELS signals recorded at different electron velocities. For slower electrons, the lowest energy modes are red-shifted further compared to high velocity electrons. In the limit as the electron velocity approaches the speed of light, however, the transformed LS modal peak occurs at lower energy than the peak in EELS. The coefficients $\chi_{l}^{L S}$ and $\chi_{l}^{E E L S}$ also depend on the impact parameter, contributing to the differences noted in Fig. 7. The energy-offset induced by the EELS and transformed LS coefficients remains a predictable and systematic modification of the underlying modes defined by the Mie coefficients and determined only by composition and geometry. In the possible application of this method to comparing EELS and LS in other geometries, the velocity, spatial, and energy dependence of modes will necessarily have to be assessed separately. The singular trend in relative probabilties in Fig. 5(b) demonstrates the plausible comparison of LS and EELS even for sphere sizes (ca. $50 \mathrm{~nm}$ ) with non-zero energy offsets.

The polarization of the incident light wave also plays an important role in comparing LS and EELS. In the case of the sphere, the symmetry of the particle allows for a simple approximation that the predominant contribution to the electron excitation is due to electric field components parallel to the shortest distance from the center of the sphere to the electron trajectory. This approximation adequately resembles the electric field of a polarized plane 
wave for impact parameters coincident with the axis of polarization. Figure 9(a) illustrates these geometries. The expression for the z-component of the electric field outlined in Eq. 1826 does not specify a particular subset of trajectories. The selection of trajectories coincident with the polarization of the field matches the most physically appropriate configuration and yields consistent comparisons for sphere composition, size, and impact parameter.

The critical distinction between the fields near an electron and those of a polarized plane wave is the spatial variance or invariance of the polarization. Near an electron, the field polarizations vary significantly in $(x, y, z)$ (see Fig. 9). In the case of a plane wave, the polarization is invariant with respect to coordinate. Notably, the spatial variance of the field near an electron is not identical to a superposition of multiple polarizations. The superposition of two antiparallel polarizations of light, for example, would in fact cancel, whereas the antiparallel field polarizations near an electron do not coincide in space. Such spatial separation of field polarization can be used to model non-dipole modes in LS, ${ }^{43}$ but is an artificial computational construct and does not model LS excitations. Consequently, a single polarization or set of polarizations must be selected in LS for comparison with EELS. The presented method enables various choices in principle, possibly selections corresponding to polarizations in experimental or application configurations. Transformed LS then gives $\Gamma^{L S}$ for optically accessible modes. Here, we note that for external electron trajectories, there exists a single spatially invariant component of the electric field interacting with the metal sphere. The corresponding plane wave polarization parallel to this field component is depicted in Fig. 9(a).

For comparing the entire signal recorded in an EELS map, the polarization of the LS electric field must be rotated to match the radial excitation condition. Figure 9(b)-(c) presents a calculated EELS map for a $10 \mathrm{~nm} \mathrm{Al} \mathrm{sphere} \mathrm{and} \mathrm{the} \mathrm{corresponding} \mathrm{matching}$ transformed LS map accounting for $360^{\circ}$ polarization rotation, each at the energy of the peak in the $l=1$ mode. For the case of a sphere, such a polarization correction is computationally trivial as all radial lines are equivalent by symmetry but serves to demonstrate an approach to the issue of light polarization. Given the description of the relative weighting factors outlined in Sec. IV, each point in the entire data cube $\Gamma^{L S}\left(\mathbf{R}_{\mathbf{0}}, \omega\right)$ can be scaled quantitatively to the EELS signal.

The consideration of polarization in relating EELS and LS plasmon responses is critical for connecting observations in EELS to plasmonic devices and technologies driven by light 
excitation. EELS fails to reproduce the polarization dependent excitation of plasmonic particles and prospective device components in a straight-forward manner, ${ }^{23}$ and so including fair polarization comparisons with LS will prove important in extending transformed LS comparisons to more complex geometries. Even in the case of a sphere, where polarization dependencies in LS are minimal, EELS mapping does not reveal the dipolar quality of the $l=1$ mode (see Fig. 9). Accounting for the opposing fields near an electron may be necessary for appropriate transformation of LS for comparison with EELS in geometries that give rise to plasmonic hot spots in LS but are not directly detected by EELS (e.g., particle dimers). ${ }^{17,24,44}$ Several approaches to symmetry breaking to make such modes accesible to light have been reported. ${ }^{14,43}$ For many geometries and electron trajectories, the scattered electric fields for LS and EELS will be similar, ${ }^{29}$ encouraging the comparison of transformed LS and EELS in other nanoparticle shapes.

Moreover, the direct comparison of LS and EELS will allow for clear distinction between bright and dark modes. Non-dipole modes, while generally considered dark, are excited by light when retardation effects are accounted for, as in the case of the sphere, or by substrateinduced symmetry breaking. ${ }^{43}$ Other dark modes such as disk breathing modes ${ }^{15}$ and toroidal modes ${ }^{16}$ depend uniquely on the spatially variant field polarization of an electron beam and cannot be excited readily by a plane wave. The presented method, by providing a direct comparison of LS and EELS, enables the separation of the origins of such dark and bright modes.

Geometries of reduced symmetry present challenges not only due to the additional LS polarization considerations, but also due to the absence of analytical modal decompositions of the electric field. Semi-analytical approaches such as T-matrix methods ${ }^{27,45}$ may present an alternative for spheroidal geometries. Small particles exhibiting predominantly dipolar excitations may be adequately compared without explicit modal decomposition of the field given sufficient resolution of the electric field contributions in the energy dimension. The successful application of non-retarded expressions (Eq. 8) for the analysis of LS and EELS by modal decomposition may invite similar approaches to the comparison of LS and EELS using total field methods where analytical modal decompositions are not possible. An analogous method of comparison might insert an LS field into the general expression for EELS probability (Eq. 2). Such a comparison has not been explored here in favor of LS and EELS comparisons for each mode. The modal decomposition approach is not limited to particular 
geometries and suggests that geometric modes underpin surface plasmon excitation probabilities generally for various excitations. ${ }^{30}$ A total field approach would allow for comparison of LS and EELS using discrete dipole approximation methods. ${ }^{28,29,46}$ Quasistatic eigenmode decompositions outlined in work by Boudarham and Kociak ${ }^{30}$ and implemented using the boundary element method ${ }^{26,32,47}$ may also hold promise for modal decomposition comparisons in other geometries. The success of numerical evaluation of the Fourier transform of the electric field along a trajectory for spheres supports the extension of the approach to other discretized methods. Work on developing transformed LS comparisons for less symmetric geometries is underway in our group.

\section{CONCLUSIONS}

Surface plasmon resonances probed by EELS have been compared directly to excitations by light by performing a modal decomposition and Fourier transformation of the component of the electric field or potential along the trajectory. This method offers quantitative relationships between surface plasmon modes excited by electrons and light. Across a wide range of plasmon energies $\omega_{P}$, the relative weighting of modes in EELS and transformed LS is described by a single function. For small spheres, the approximation of the Fourier transform as a projection of potential may also serve to compare LS and EELS.

In comparing EELS and transformed LS, the excited volume for an electron or a plane wave gave rise to key distinctions in relative weighting of surface plasmon modes. The comparison of transformed LS and EELS is simple in the case of small spheres where the excited volume is similar. For large spheres, the excited volume increases for LS but proportionally decreases for EELS. The variation in excited volume for electron excitation is further manifest in variation in the impact parameter dependence of EELS with size.

As a demonstration of the comparison of polarized plane wave illumination and EELS, transformed LS mapping of a sphere outlined requirements for evaluating polarization and selecting trajectories particular to the particle geometry. Tenable methods for the comparison of LS and EELS simulations and experimental data will allow for enhanced validation and assessment of the technologically-relevant LS behaviour of plasmonic nanoparticles by EELS. Further application of this direct link between LS and EELS may enable the estimation of such properties as the near field enhancement directly from EELS data. 


\section{ACKNOWLEDGMENTS}

S.M.C. acknowledges the support of a Gates Cambridge Scholarship. The research leading to these results has received funding from the European Research Council under the European Union's Seventh Framework Programme (FP7/2007-2013)/ERC grant agreement

291522-3DIMAGE. P.A.M. also acknowledges funding from the European Union's Seventh Framework Programme under a contract for an Integrated Infrastructure Initiative (Reference 312483-ESTEEM2).

* smc204@cam.ac.uk

$\dagger$ pam33@cam.ac.uk

1 P. Tiemeijer, Ultramicroscopy 78, 53 (1999).

2 M. Terauchi, M. Tanaka, K. Tsuno, and M. Ishida, J. Microsc. 194, 203 (1999).

3 E. Essers, M. Matijevic, G. Benner, R. Höschen, W. Sigle, and C. Koch, Microsc. Micronanal. 13, 18 (2007).

4 S. Nie and S. R. Emory, Science 275, 1102 (1997).

5 J. N. Anker, W. P. Hall, O. Lyandres, N. C. Shah, J. Zhao, and R. P. V. Duyne, Nat. Mater. 7, 442 (2008).

6 V. E. Ferry, L. A. Sweatlock, D. Pacifici, and H. A. Atwater, Nano Lett. 8, 4391 (2008).

7 K. R. Catchpole and A. Polman, Opt. Express 16, 21793 (2008).

8 J. S. Biteen, D. Pacifici, N. S. Lewis, and H. A. Atwater, Nano Lett. 5, 1768 (2005).

9 K. Okamoto, I. Niki, A. Shvartser, Y. Narukawa, T. Mukai, and A. Scherer, Nat. Mater. 3, 601 (2004).

10 S. Mukherjee, F. Libisch, N. Large, O. Neumann, L. V. Brown, J. Cheng, J. B. Lassiter, E. A. Carter, P. Nordlander, and N. J. Halas, Nano Lett. 13, 240 (2013).

11 W. H. Hung, M. Aykol, D. Valley, W. Hou, and S. B. Cronin, Nano Lett. 10, 1314 (2010).

12 J. A. Schuller, E. S. Barnard, W. Cai, Y. C. Jun, J. S. White, and M. L. Brongersma, Nat. Mater. 9, 193 (2010).

13 E. Verhagen, R. de Waele, L. Kuipers, and A. Polman, Phys. Rev. Lett. 105, 223901 (2010).

14 M.-W. Chu, V. Myroshnychenko, C. H. Chen, J.-P. Deng, C.-Y. Mou, and F. J. García de 
Abajo, Nano Lett. 9, 399 (2009).

15 F.-P. Schmidt, H. Ditlbacher, U. Hohenester, A. Hohenau, F. Hofer, and J. R. Krenn, Nano Lett. 12, 5780 (2012).

16 B. Ögüt, N. Talebi, R. Vogelgesang, W. Sigle, and P. A. van Aken, Nano Lett. 12, 5239 (2012).

17 B. S. Guiton, V. Iberi, S. Li, D. N. Leonard, C. M. Parish, P. G. Kotula, M. Varela, G. C. Schatz, S. J. Pennycook, and J. P. Camden, Nano Lett. 11, 3482 (2011).

18 J. Nelayah, M. Kociak, O. Stéphan, N. Geuquet, L. Henrard, F. J. García de Abajo, I. PastorizaSantos, L. M. Liz-Marzán, and C. Colliex, Nano Lett. 10, 902 (2010).

19 S. Mazzucco, N. Geuquet, J. Ye, O. Stéphan, W. Van Roy, P. Van Dorpe, L. Henrard, and M. Kociak, Nano Lett. 12, 1288 (2012).

20 M. Bosman, G. R. Anstis, V. J. Keast, J. D. Clarke, and M. B. Cortie, ACS Nano 6, 319 (2012).

21 J. Zuloaga and P. Nordlander, Nano Lett. 11, 1280 (2011).

22 D. Rossouw, M. Couillard, J. Vickery, E. Kumacheva, and G. A. Botton, Nano Lett. 11, 1499 (2011).

23 D. Rossouw and G. A. Botton, Phys. Rev. Lett. 110, 066801 (2013).

24 U. Hohenester, H. Ditlbacher, and J. R. Krenn, Phys. Rev. Lett. 103, 106801 (2009).

25 F. J. García de Abajo and M. Kociak, Phys. Rev. Lett. 100, 106804 (2008).

26 U. Hohenester and A. Trügler, Comput. Phys. Commun. 183, 370 (2012).

27 C. Matyssek, V. Schmidt, W. Hergert, and T. Wriedt, Ultramiscroscopy 117, 46 (2012).

28 N. Geuquet and L. Henrard, Ultramicroscopy 110, 1075 (2010).

29 N. W. Bigelow, A. Vaschillo, V. Iberi, J. P. Camden, and D. J. Masiello, ACS Nano 6, 7497 (2012).

30 G. Boudarham and M. Kociak, Phys. Rev. B 85, 245447 (2012).

31 R. H. Ritchie, Phys. Rev. 106, 874 (1957).

32 F. J. García de Abajo, Rev. Mod. Phys. 82, 209 (2010).

33 N. Zabala and A. Rivacoba, Phys. Rev. B 48, 14534 (1993).

34 T. L. Ferrell and P. M. Echenique, Phys. Rev. Lett. 55, 1526 (1985).

35 A. Rivacoba, N. Zabala, and J. Aizpurua, Prog. Surf. Sci. 65, 164 (2000).

36 F. J. García de Abajo, Phys. Rev. B 59, 3095 (1999).

37 C. F. Bohren and D. R. Huffman, Absorption and scattering of light by small particles (John 
Wiley \& Sons, New York, 1983).

38 R. Fuchs and K. L. Kliewer, J. Opt. Soc. Am. 58, 319 (1968).

39 J.-P. Schäfer, Implementierung und Anwendung analytischer und numerischer Verfahren zur Lösung der Maxwellgleichungen für die Untersuchung der Lichtausbreitung in biologischem Gewebe, Ph.D. thesis, Universität Ulm. Fakultät für Naturwissenschaften (2011).

40 E. J. Zeman and G. C. Schatz, J. Phys. Chem. 91, 634 (1987).

41 P. M. Echenique, A. Howie, and D. J. Wheatley, Philosophical Magazine Part B 56, 335 (1987).

42 M. Achèche, C. Colliex, H. Kohl, A. Nourtier, and P. Trebbia, Ultramicroscopy 20, 99 (1986).

43 S. Zhang, K. Bao, N. J. Halas, H. Xu, and P. Nordlander, Nano Lett. 11, 1657 (2011).

44 N. Mirsaleh-Kohan, V. Iberi, P. D. Simmons, N. W. Bigelow, A. Vaschillo, M. M. Rowland, M. D. Best, S. J. Pennycook, D. J. Masiello, B. S. Guiton, and J. P. Camden, J. Phys. Chem. Lett. 3, 2303 (2012).

45 M. I. Mishchenko, J. W. Hovenier, and L. D. Travis, Light scattering by nonspherical particles: theory, measurements, and applications (Academic Press, San Diego, 2000).

46 P. J. Flatau and B. T. Draine, J. Opt. Soc. Am. A 11, 1491 (1994).

47 F. J. García de Abajo and A. Howie, Phys. Rev. B 65, 115418 (2002). 

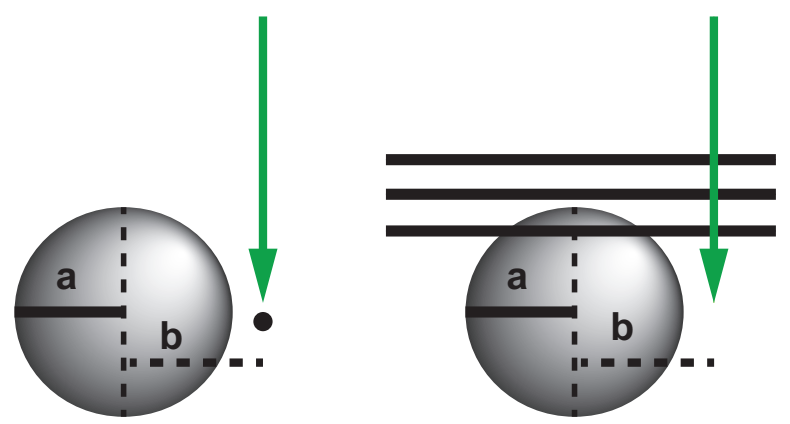

FIG. 1. (Color online) Illustration of the definition of the radius, $a$, and impact parameter, $b$, for electron (left) and plane wave (right) excitation of a plasmonic metal nanosphere.
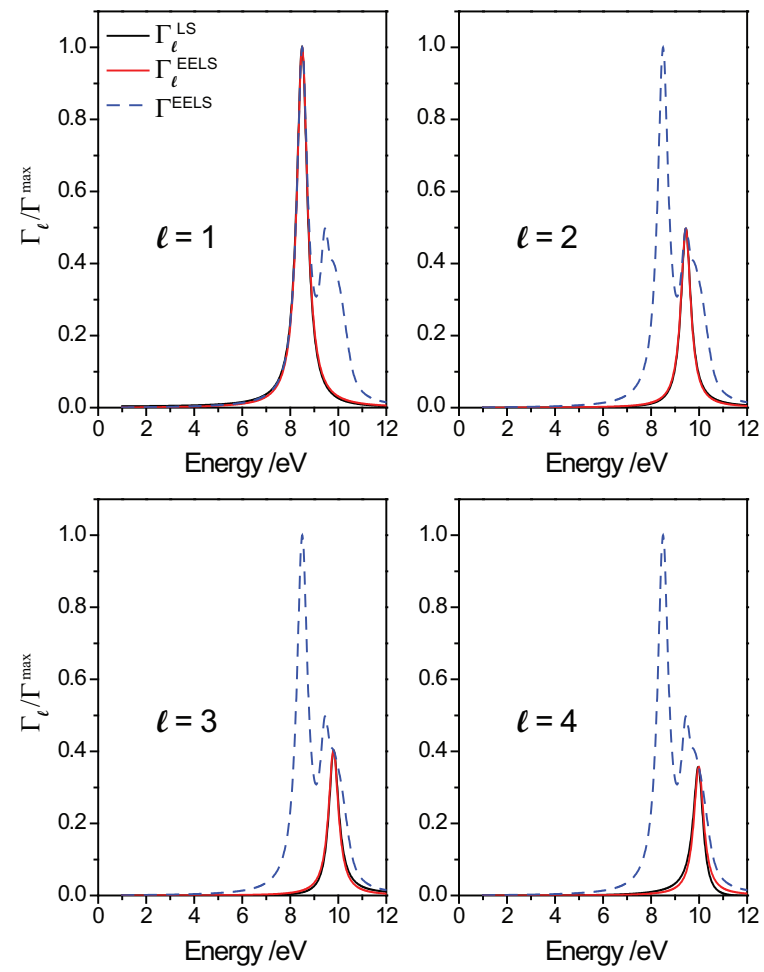

FIG. 2. (Color online) Normalized modes for $l=1-4$ calculated by the transformed LS probability $\Gamma_{l}^{L S}$ (solid black, Eq. 30) and the analytical EELS probability $\Gamma_{l}^{E E L S}$ (solid red, Eq. 11) for a 10 $\mathrm{nm}$ diameter $\mathrm{Al}$ sphere modeled with a Drude dielectric function parameterized according to $\hbar \omega_{P}$ $=15 \mathrm{eV}, \hbar \Gamma=0.5 \mathrm{eV}$ (following Ref. 32). The total EELS probability $\Gamma^{E E L S}$ (dashed blue) is also shown for reference. Impact parameter $b=1.1 a$. 

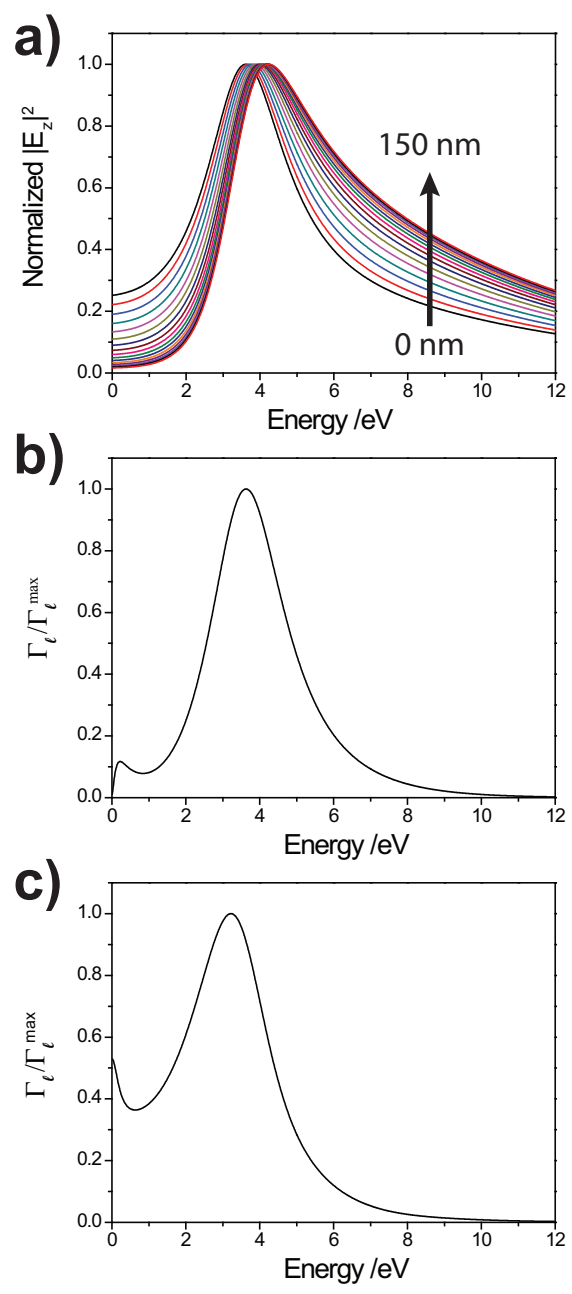

FIG. 3. (Color online) (a) The electric field intensity $\left|E_{z}\right|^{2}$ for mode $l=1$ plotted for planes 0 $\mathrm{nm}$ to $150 \mathrm{~nm}$ above a $100 \mathrm{~nm}$ diameter $\mathrm{Al}$ sphere (increments of $10 \mathrm{~nm}$ shown). The heights are given from the plane containing the uppermost point of the particle. (b) The corresponding EELS and (c) transformed LS probabilities for the dipolar mode $(l=1)$ are presented for comparison. Impact parameter $b=1.1 a$. 

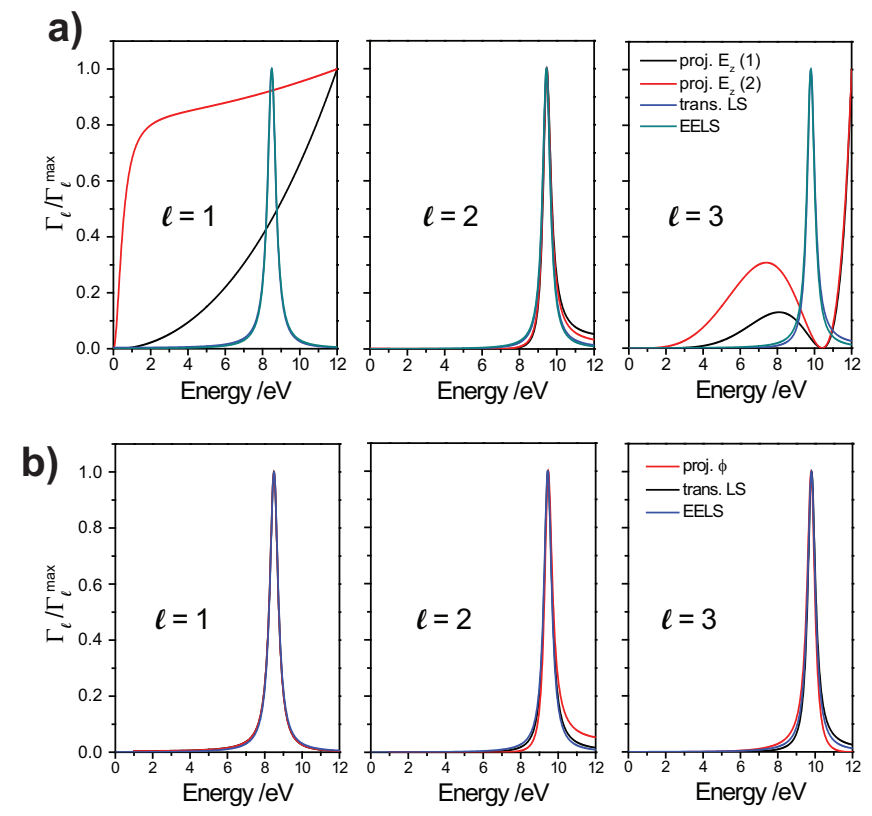

FIG. 4. (Color online) Comparison of EELS and transformed LS to alternative integrals of (a) field $E_{z}$ and (b) potential $\phi$ for modes $l=1-3$ for a $10 \mathrm{~nm} \mathrm{Al}$ sphere. Comparisons are plotted as $\Gamma_{l}$ normalized to the peak value $\Gamma_{l}^{\max }$. (a) (1) projection of $E_{z}$ given by Eq. 38 (2) projection adjusted by $\omega^{-2}$ given by Eq. 39. The transformed LS and EELS modes follow Eq. 30 and Eq. 11, respectively. (b) Projection of $\phi$ given by Eq. 40, transformed LS calculated from the Fourier transform of the electric potential, and the analytical EELS probability. Impact parameter $b=1.1 a$. 

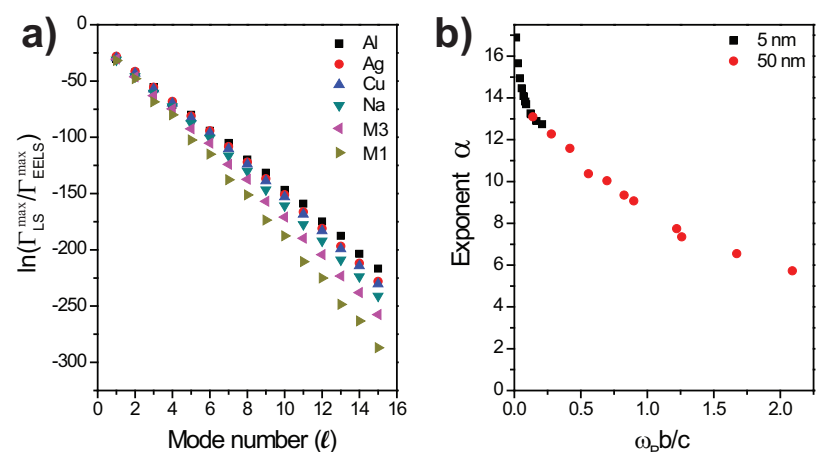

FIG. 5. (Color online) (a) Ratios of transformed LS and EELS peak values $\Gamma^{\max }$ for modes $l=1-15$ for $5 \mathrm{~nm}$ diameter spheres of $\mathrm{Al}, \mathrm{Ag}, \mathrm{Na}$, and $\mathrm{Cu}$ and additional hypothetical metals modeled by Drude dielectric functions. Metals were parameterized according to Ref. 32, 40. M3 and M1 refer to metals modeled with $\hbar \omega_{P}=3 \mathrm{eV}$ and $\hbar \omega_{P}=1 \mathrm{eV}$, respectively, and $\hbar \Gamma=0.1 \mathrm{eV}$. (b) The exponent $\alpha$ for the rate of decay in terms of the plasmon energy, $\omega_{P}$, for $5 \mathrm{~nm}$ and $50 \mathrm{~nm}$ diameter spheres $(b=1.1 a)$. Modes $l=1-6$ were used for fitting to avoid numerical imprecision in higher order modes due to their low excitation probability. 

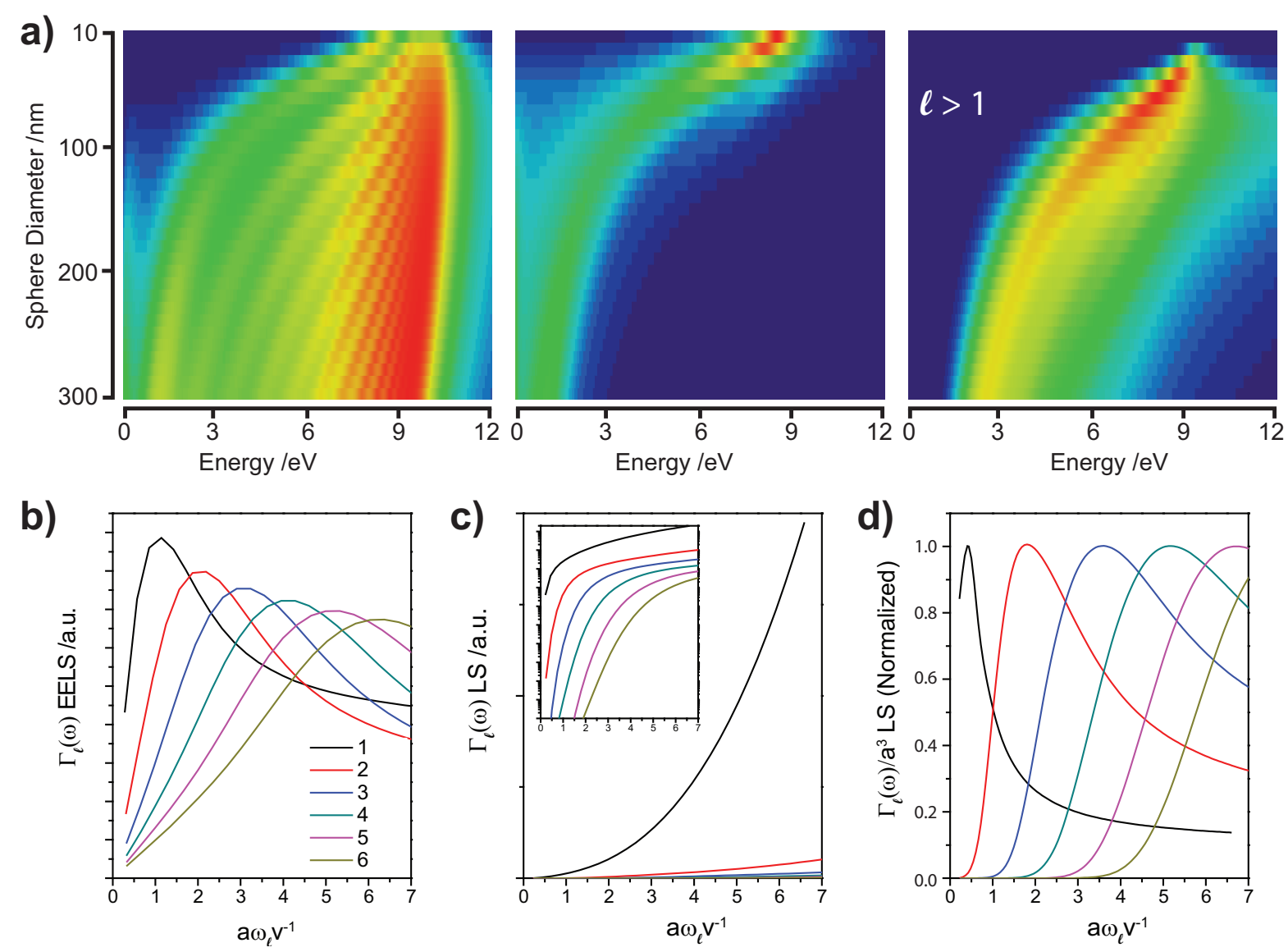

FIG. 6. (Color online) (a) Size dependence of (left) EELS and (center) volume-adjusted transformed LS for 10-300 nm diameter Al spheres. The size dependence of volume-adjusted transformed LS modes $l>1$ (right) is re-plotted separately. Probabilities are plotted on a logarithmic color scale. (b) EELS probability, (c) transformed LS, and (d) volume-adjusted transformed LS for modes $l=1-6$ as a function of reduced radius $a \omega_{l} / v$. In (c) the transformed LS modal $\Gamma_{l}$ is replotted on a logarithmic scale to visualize the low intensity modes (inset). Impact parameter set to grazing incidence such that $b=a$ following Ref. 35 . 

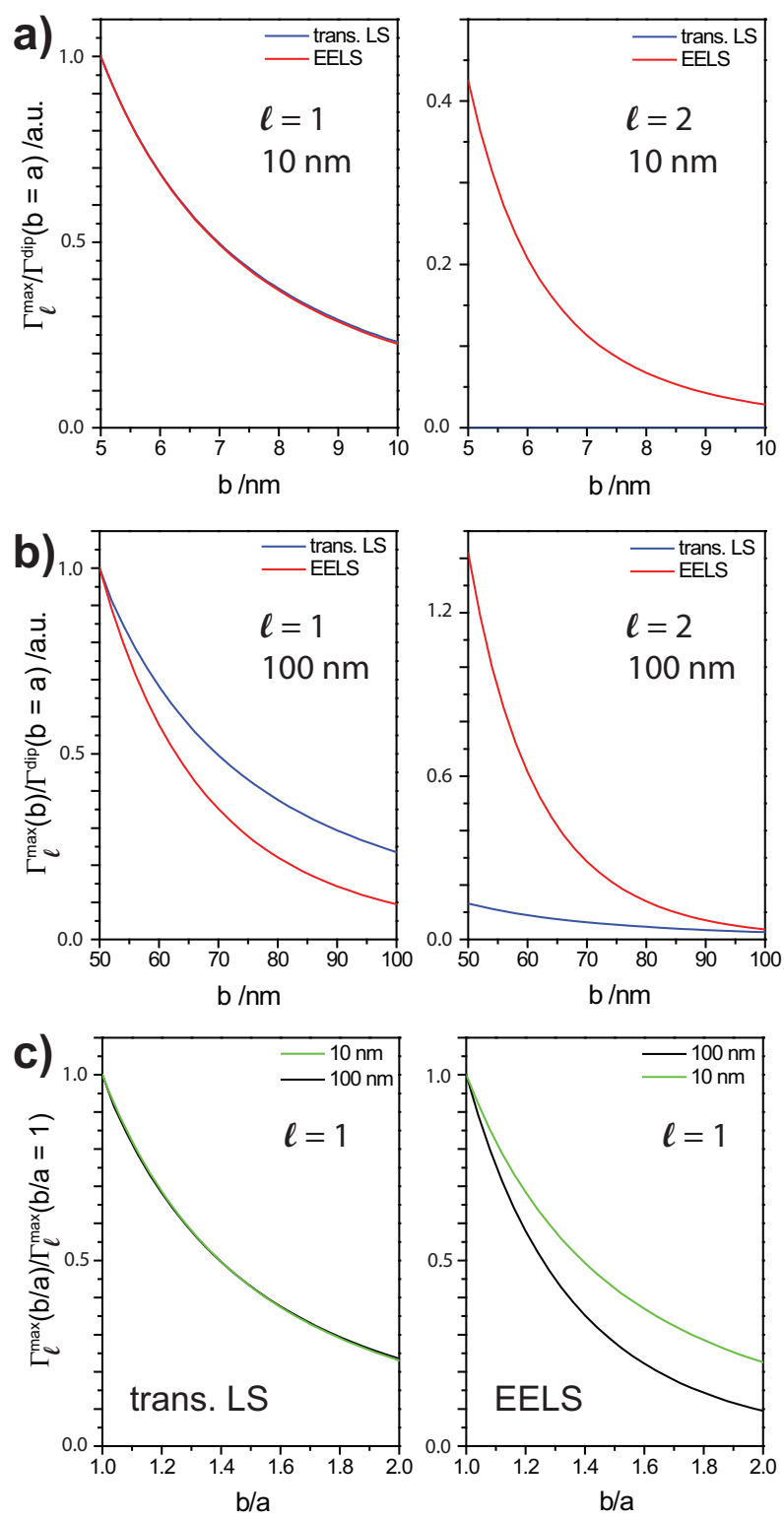

FIG. 7. (Color online) Impact parameter dependence of modal probability maximum $\Gamma^{\max }$ for transformed LS (blue) and EELS (red) for (a) $10 \mathrm{~nm}$, (b) 100 diameter Al spheres. The maxima in the transformed LS probability $\Gamma^{\max }$ were normalized to the maximum in the EELS probability at the surface of the sphere $(b=a)$ for the $l=1$ mode $\left(\Gamma^{\text {dip }}\right)$. (c) Relative impact parameter dependence of $l=1$ probability for $10 \mathrm{~nm}$ (green) and $100 \mathrm{~nm}$ (black) Al spheres for transformed LS and EELS. 


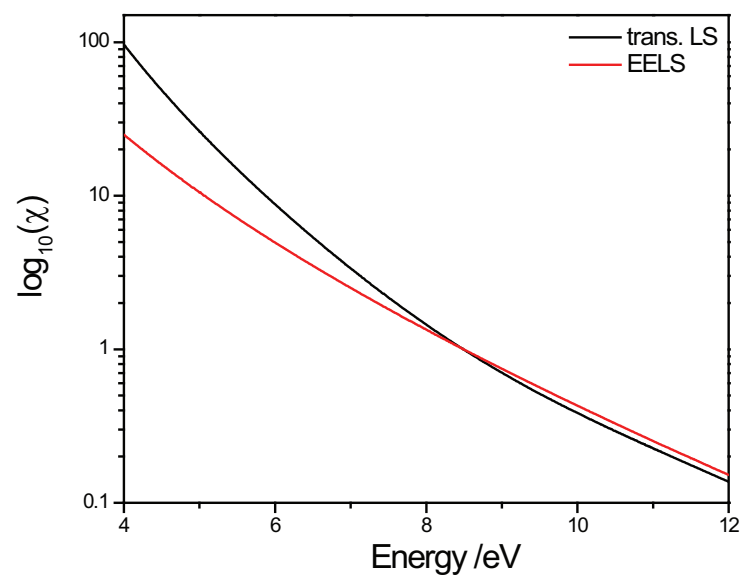

FIG. 8. (Color online) Coefficients, $\chi_{l}$, following Eq. 35-36 for dipolar $(l=1)$ surface plasmon mode excitation by (black) a plane wave of light and (red) a $300 \mathrm{kV}$ electron. Coefficients were normalized at the energy corresponding to the maximum in the $l=1$ mode for the $10 \mathrm{~nm}$ diameter $\mathrm{Al}$ spheres in Fig. 1. Impact parameter $b=30 \mathrm{~nm}$.
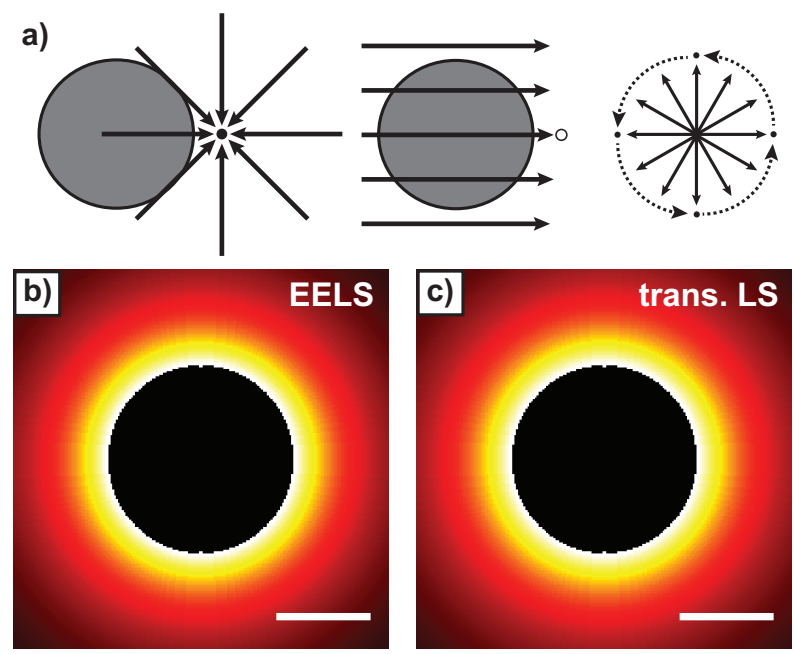

FIG. 9. (Color online) (a) Diagrams illustrating the electric field directions for (left) EELS and (center) LS. The direction of the electron and corresponding light propagation is into the page. To account for the position dependence of EELS excitation, the field polarization is rotated for calculating transformed LS probabilities (right). Maps of the dipolar $(l=1)$ surface plasmon excitation for external trajectories in (b) EELS and (c) transformed LS for a $10 \mathrm{~nm}$ diameter $\mathrm{Al}$ sphere. Scale bars are $5 \mathrm{~nm}$. 